\title{
Cancer Model Development Center
}

National Cancer Institute

\section{Source}

National Cancer Institute. Cancer Model Development Center. NCI Thesaurus. Code C156876.

National Cancer Institute-funded contributors to the Human Cancer Genome Initiative that are tasked with producing next-generation cancer models from clinical samples. 